\title{
MÉTODO PARA VALIDAÇÃo DA PREDIÇÃO DA PERDA DE CARGA EM CICLONES COM BASE EM RESULDADOS DE CFD
}

\author{
E. BALESTRIN*, R. K. DECKER, J. C. S. C. BASTOS, H. F. MEIER \\ Universidade Regional de Blumenau, Departamento de Engenharia Química \\ e-mail: evandrobalestrin@hotmail.com
}

\begin{abstract}
RESUMO
Ciclones são equipamentos vastamente utilizados para separação de partículas sólidas suspensas em uma fase gasosa. Tal fase gasosa serve como transporte para as partículas sólidas, sendo assim a principal responsável pelo comportamento giratório e de alta intensidade de turbulência do escoamento multifásico observado em ciclones. Esses dispositivos são avaliados com base em dois parâmetros de desempenho, a eficiência de coleta e a perda de carga, sendo o último relacionado ao custo operacional. Portanto, o presente estudo tem como objetivo analisar a forma mais apropriada de se aferir a perda de carga em ciclones através de resultados de CFD, a fim de validar tais predições. Neste trabalho, realizou-se experimentos físicos e simulações numéricas para predizer a perda de carga do escamento monofásico em uma faixa de velocidade entre 8 e $16 \mathrm{~m} / \mathrm{s}$. As simulações numéricas foram efetuadas utilizando-se uma modelagem RANS (Reynolds Averaged Navier-Stokes) e o modelo RSM (Reynolds Stress Model) para fechamento da turbulência. Dos resultados simulados, avaliou-se a perda de carga através de dois métodos, a diferença entre a pressão média em planos na entrada e na saída e a diferença entre uma média em quatro pontos definidos na entrada e na saída do ciclone. Comparando os dois métodos com os dados experimentais, observou-se que os resultados da média nos pontos ficaram mais próximos dos resultados experimentais frente ao método com a média no plano. Sendo assim, mostrando-se a forma mais eficiente para a validação da perda de carga.
\end{abstract}

\section{INTRODUÇÃO}

Ciclones são dispositivos utilizados para separação gás-sólido, bastante empregados para coletar partículas superiores a $5 \mu \mathrm{m}$, com ênfase nas indústrias de refino de petróleo, dentro das unidades de FCC (Fluid Catalytic Cracking), em industrias cimenteiras e no controle de poluição. São equipamentos de simples construção e suportam variadas condições de operação, como altas temperaturas e pressão.

$\mathrm{O}$ ciclone tem como princípio de funcionamento, a separação do sólido como consequência do forte escoamento giratório que juntamente com a ação da força centrifuga desloca as partículas em direção às paredes do ciclone, e da grande intensidade de turbulência que acarreta na reversão do escoamento da fase gasosa. Os engenheiros estão geralmente interessados em dois parâmetros, quando se trata da avaliação de projetos e performance em ciclones, a eficiência de coleta e a perda de carga. A eficiência de coleta é definida como a razão entre a quantidade de sólido coletado e a quantidade de sólido alimentado, já a perda de carga é a diferença entre a pressão na entrada e na saída do ciclone. A última está relacionada ao consumo energético, ou seja, quanto maior a perda de carga no ciclone maior o consumo. 
Inúmeros estudos em ciclones já foram realizados a fim de avaliar a perda de carga experimentalmente, trabalhos que vão desde os realizados por Yuu et al. (1978), que avaliaram a redução da perda de carga em relação ao carregamento de sólido em um ciclone convencional, até Funk (2015) que analisou a redução da perda de carga devido modificações na saída do gás. No entanto, métodos de avaliações experimentais requerem um alto investimento, sendo assim, os códigos de CFD têm sido uma alternativa para tais avaliações, tendo em vista o grande potencial em predizer o escoamento característico dos ciclones. Potencial esse, apresentados em trabalhos como Noriler et al. (2004), Gimbun et al. (2005) e Chu et al. (2011).

Entretanto, na avaliação dos resultados simulados e na validação dos mesmos a partir de dados experimentais, deve-se ter alguns cuidados. Portanto, o presente estudo tem como objetivo analisar a forma mais apropriada de se aferir a perda de carga em ciclones através de resultados de CFD, a fim de validar tais predições.

\section{MATERIAIS E MÉTODOS}

O materiais e métodos foram separados em dois tópicos, aqueles utilizados para realização dos experimentos físicos e os utilizados nas simulações numéricas.

\subsection{Experimentos físicos}

Os experimentos físicos foram realizados em unidade experimental, conforme ilustrada na Figura 1. A configuração utilizada para obtenção dos dados experimentais relacionados ao escoamento monofásico, consiste em um conjunto de tubulações de admissão da fase gasosa (01), que passa pelo ciclone de acrílico (07) e pelo filtro manga (09) antes de sair através do exaustor (10).

Figura 1 - Unidade experimental: 01 Dutos; 02 Tubo de Pitot; 03 Medidor diferencial de pressão; 04 Medidor de pressão; 05 Medidor de temperatura; 06 Sistema de alimentação de sólidos; 07 Ciclone; 08 Caixa coletora de sólido; 09 Filtro Manga; 10 Exaustor.

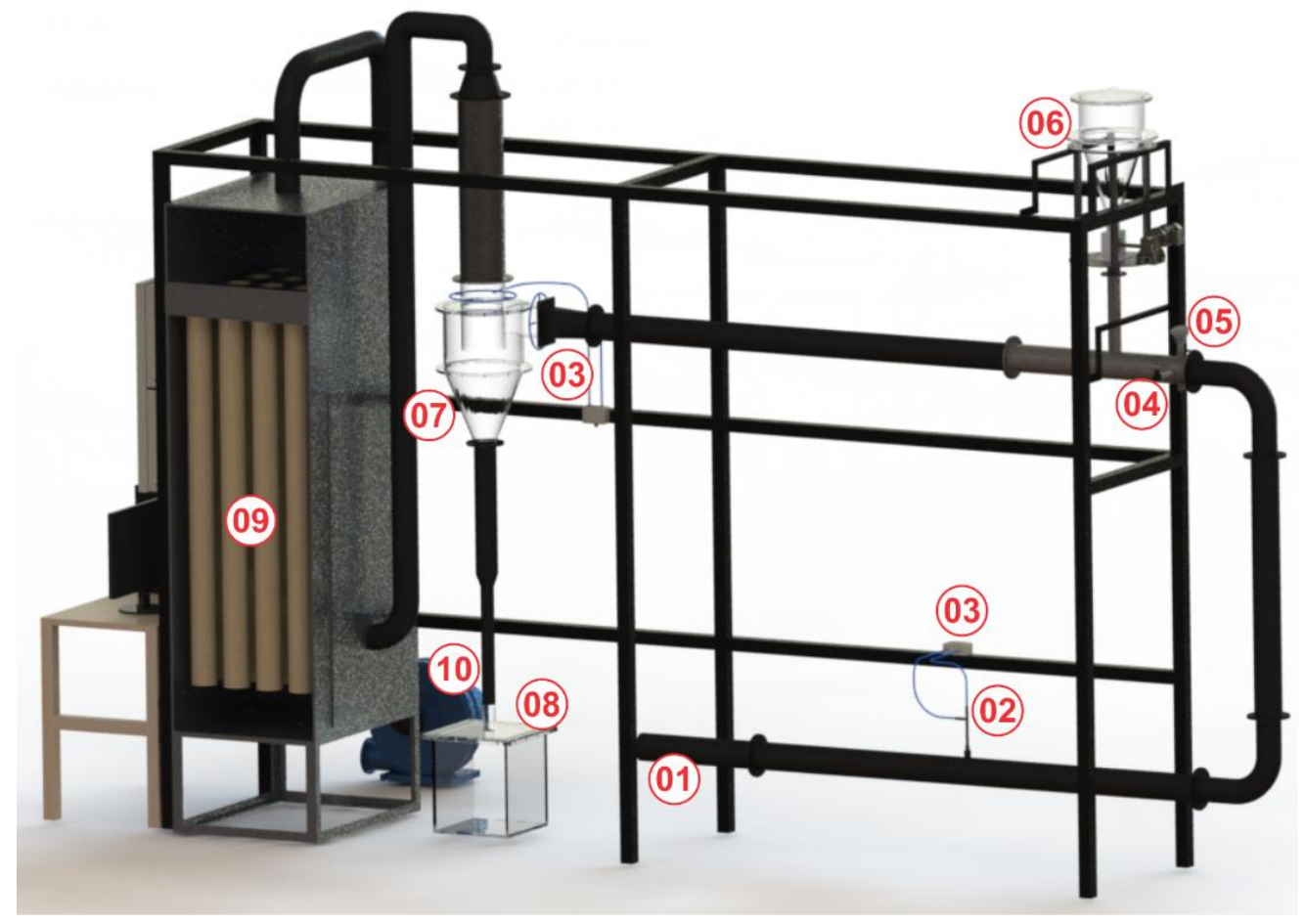


Para medidas de perda de carga, tem-se um medidor diferencial de pressão (03) acoplado ao ciclone, que obtêm a diferença entre as medidas de pressão na entrada e na saída do equipamento.

Sendo assim, realizou-se medições de perda de carga para o escoamento monofásico variando-se a velocidade de entrada do ciclone em uma faixa de 8 a $16 \mathrm{~m} / \mathrm{s}$. Os experimentos de perda de carga foram repetidos dez vezes para cada velocidade, para com isso avaliar e quantificar as incertezas experimentais. A velocidade na entrada é controlada por um CLP (Controlador Lógico Programado), e medida através de um tubo de Pitot (02).

\subsubsection{Geometria do ciclone}

Ciclones são equipamentos compostos basicamente por quatro regiões, a região de entrada, do corpo cilíndrico, do corpo cônico e do tubo de imersão (vortex finder). Utilizou-se para realização das experimentações físicas um ciclone em acrílico e com entrada tangencial. A ilustração e as dimensões do ciclone utilizado encontram-se na Figura 2.

Figura 2 - Ciclone em acrílico e suas medidas em milímetros.
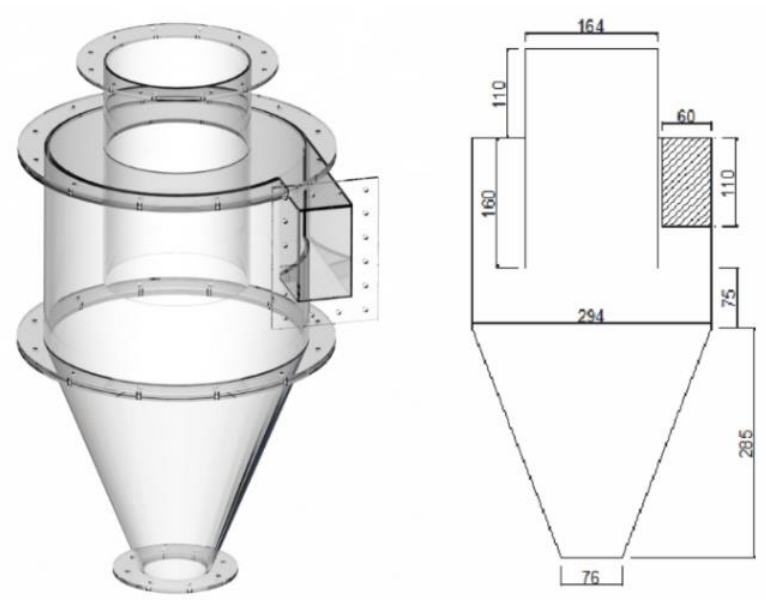

\subsection{Simulações numéricas}

As simulações numéricas foram todas efetuadas em um código comercial, Fluent 14 da ANSYS. Este utiliza o método de volumes finitos como método de discretização das equações diferenciais do modelo matemático.

As configurações realizadas para as simulações numéricas foram: modelo de turbulência "RSM-SSG", acoplamento pressão velocidade "SIMPLEC" e discretização espacial da pressão por algoritmo "PRESTO", do movimento com esquema "Second Order Upwind", e da energia cinética turbulenta e da taxa de energia cinética turbulenta com "First Order Upwind", além da formulação implícita no tempo.

As simulações numéricas seguem o mesmo planejamento e geometria (Figura 3) dos experimentos físicos, de forma que as condições de velocidade na entrada do duto utilizadas foram $7 ; 8,75 ; 10,5 ; 12,25$ e $14 \mathrm{~m} / \mathrm{s}$, que equivalem aos $8 ; 10 ; 12 ; 14$ e $16 \mathrm{~m} / \mathrm{s}$ na entrada do ciclone.

Figura 3 - Geometria da unidade experimental utilizada nas simulações.

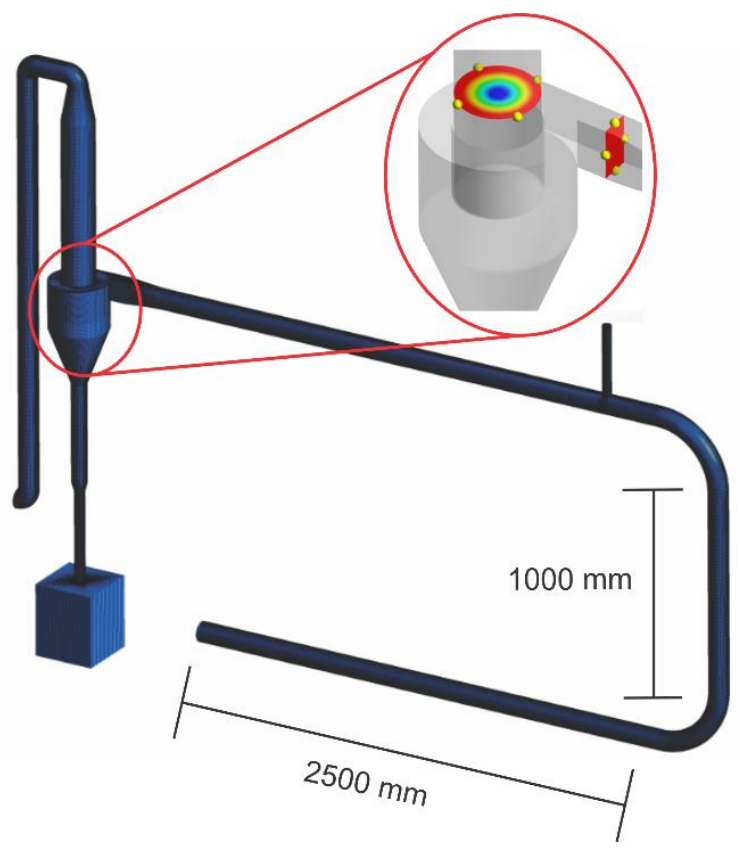

A Figura 3 mostra a localização das medidas de pressão na entrada e na saída do ciclone, afim de calcular a perda de carga dos resultados numéricos. Também apresenta a diferença entre os dois métodos utilizados para aferir a perda de carga dos resultados 
simulados, diferença das pressões medidas da média nos planos e nos pontos da entrada e saída do ciclone.

\subsubsection{Quantificação da incerteza numérica}

Segundo Freitas (2002) a incerteza pode ser definida como sendo a quantidade estimada ou porcentagem em que um valor observado ou calculado pode diferir do valor verdadeiro.

A incerteza numérica foi obtida através do estimador GCI (Grid Convergence Index). Este método é baseado em um estimação do erro de refinamento de malha derivado da teoria generalizada da extrapolação de Richardson. Para que seja possível utilizar este método, é necessário duas soluções numéricas distintas, sendo a primeira solução obtida a partir de uma malha refinada $\left(f_{1}\right)$ e a segunda a partir de uma malha grosseira $\left(f_{2}\right)$ (ROACHE, 1994). A equação utilizada para o cálculo do GCI foi:

$$
\mathrm{GCI}_{1}=F_{s} \frac{\left|f_{1}-f_{2}\right|}{1-r^{q}}
$$

onde $\left(F_{s}\right)$ é o fator de segurança, (r) é a razão de refinamento entre as duas malhas e (q) é a ordem formal de precisão do algoritmo.

Roache (1994) e Karimi et al. (2012) recomendam utilizar um fator de segurança $\mathrm{F}_{\mathrm{s}}=3$ para estudos desenvolvidos com duas malhas numéricas e $F_{\mathrm{s}}=1,25$ para três ou mais malhas. Os parâmetros aplicados neste estudo foram: $\mathrm{r}=1,2, \mathrm{~F}_{\mathrm{s}}=3$ and $\mathrm{q}=2$.

\section{MODELAGEM MÁTEMATICA}

A modelagem matemática aplicada nas simulações numéricas é fundamentada nas equações de Navier-Stokes, a partir do ponto de vista das médias temporais de Reynolds (RANS) e sob um enfoque Euleriano. As equações de conservação da massa e do movimento para a fase gasosa são expressas da seguinte maneira: equação de conservação da massa,

$$
\frac{\partial}{\partial t}\left(\rho_{g}\right)+\nabla \cdot\left(\rho_{g} v_{g}\right)=0
$$

equação de conservação do movimento,

$$
\begin{aligned}
& \frac{\partial}{\partial t}\left(\rho_{g} \boldsymbol{v}_{g}\right)+\boldsymbol{\nabla} \cdot\left(\rho_{g} \boldsymbol{v}_{g} \boldsymbol{v}_{g}\right) \\
& =-\boldsymbol{\nabla} \cdot\left(\boldsymbol{T}_{g}^{e f f}\right)+\rho_{g} \boldsymbol{g}-\boldsymbol{\nabla} p
\end{aligned}
$$

O subscrito "g" indica a fase gasosa e o tensor efetivo pode ser expresso como sendo a soma do tensor de Reynolds turbulento $\left(\mathbf{T}_{g}^{t}\right) \mathrm{e}$ o tensor molecular $\left(\mathbf{T}_{\mathrm{g}}\right)$ :

$$
\boldsymbol{T}_{g}^{e f f}=\boldsymbol{T}_{g}^{t}+\boldsymbol{T}_{g}
$$

O modelo RSM (Reynolds Stress Model) foi utilizado para realizar o fechamento da turbulência:

$$
\begin{aligned}
& \frac{\partial}{\partial \mathrm{t}}\left(\boldsymbol{T}_{g}^{t}\right)+\boldsymbol{\nabla} \cdot \mathbf{v}_{\mathrm{g}}\left(\boldsymbol{T}_{g}^{t}\right) \\
& =\mathbf{D}_{\mathbf{T}}+\mathbf{D}_{\mathbf{M}}+\mathbf{Y}+\boldsymbol{\Pi}-\frac{2}{3} \boldsymbol{\delta} \varepsilon_{\mathrm{g}}
\end{aligned}
$$

Onde a difusão turbulenta $\left(\mathbf{D}_{\mathbf{T}}\right)$ deve ser modelada como:

$$
\mathbf{D}_{\mathbf{T}}=\boldsymbol{\nabla} \cdot\left(\frac{v_{g}^{t}}{\sigma_{k}} \boldsymbol{\nabla} \mathbf{T}_{g}^{t}\right)
$$

A difusão molecular $\mathbf{D}_{\mathbf{M}}$ e a taxa de produção (Y) são expressas como:

$$
\mathbf{D}_{\mathbf{M}}=\boldsymbol{\nabla} \cdot\left(v \boldsymbol{\nabla} \mathbf{T}_{g}^{t}\right)
$$




\section{OENEMP ${ }^{x}$

$$
\mathbf{Y}=-\left[\mathbf{T}_{g}^{t} \cdot\left(\boldsymbol{\nabla} \mathbf{v}_{\mathrm{g}}\right)^{\mathrm{T}}+\mathbf{T}_{g}^{t} \cdot\left(\boldsymbol{\nabla} \mathbf{v}_{\mathrm{g}}\right)\right]
$$

A taxa de deformação devido a pressão (II) utilizada foi o modelo quadrático proposto por Speziale, et al. (1991), o qual é conhecido como RSM-SSG:

$$
\begin{aligned}
& \boldsymbol{\Pi}=-\varepsilon_{\mathrm{g}}\left[\mathrm{C}_{1} \boldsymbol{b}+\mathrm{C}_{2}\left(\mathbf{b} \cdot \mathbf{b}-\frac{1}{3} \mathbf{b}: \mathbf{b} \boldsymbol{\delta}\right)\right] \\
& -C_{1}^{*} \Upsilon^{*} \boldsymbol{b}+\mathrm{k}_{g} \boldsymbol{S}\left(\mathrm{C}_{3}-C_{3}^{*} \sqrt{\mathbf{b}: \mathbf{b}}\right) \\
& +\mathrm{C}_{4} \mathrm{k}_{\mathrm{g}}\left(\boldsymbol{b} \cdot \boldsymbol{S}^{\mathrm{T}}+\boldsymbol{S} \cdot \boldsymbol{b}^{\mathrm{T}}-\frac{2}{3} \boldsymbol{b}: \boldsymbol{S} \boldsymbol{\delta}\right) \\
& +\mathrm{C}_{5} \mathrm{k}_{\mathrm{g}}\left(\boldsymbol{b} \cdot \boldsymbol{\Omega}^{\mathrm{T}}+\boldsymbol{\Omega} \cdot \boldsymbol{b}^{\mathrm{T}}\right) .
\end{aligned}
$$

Onde o tensor anisotrópico de Reynolds $(\boldsymbol{b})$, a taxa de deformação $(\mathbf{S})$ e a vorticidade $(\boldsymbol{\Omega})$, são expressos por:

$$
\begin{aligned}
& \boldsymbol{b}=\frac{\mathbf{T}_{g}^{t}}{2 \rho_{g} \mathrm{k}_{\mathrm{g}}}-\frac{1}{3} \boldsymbol{\delta} \\
& \mathbf{S}=\frac{1}{2}\left[\boldsymbol{\nabla} \mathbf{v}_{\mathrm{g}}+\left(\boldsymbol{\nabla} \mathbf{v}_{\mathrm{g}}\right)^{\mathrm{T}}\right] \\
& \boldsymbol{\Omega}=\frac{1}{2}\left[\boldsymbol{\nabla} \mathbf{v}_{\mathrm{g}}-\left(\boldsymbol{\nabla} \mathbf{v}_{\mathrm{g}}\right)^{\mathrm{T}}\right] .
\end{aligned}
$$

A energia cinética turbulenta $(\mathrm{k})$ é representada pelo traço do tensor de Reynolds e a taxa de dissipação $(\varepsilon)$ requer uma equação de conservação como:

$$
\begin{aligned}
& \frac{\partial}{\partial t}\left(\rho_{g} \varepsilon_{g}\right)+\nabla \cdot\left(\rho_{g} \mathbf{v}_{\mathbf{g}} \varepsilon_{\mathrm{g}}\right)= \\
& \boldsymbol{\nabla} \cdot\left[\left(\mu-\frac{\mu_{g}^{t}}{\sigma_{\varepsilon}}\right) \nabla \varepsilon_{g}\right]+\frac{\varepsilon_{g}}{\mathrm{k}_{g}} \rho_{g} C_{\varepsilon 1} \Upsilon^{*} \\
& -\frac{\varepsilon_{g}{ }^{2}}{\mathrm{k}_{g}} \rho_{g} \mathrm{C}_{\varepsilon 2} .
\end{aligned}
$$

A viscosidade turbulenta $\left(\mu_{g}^{t}\right)$ é representada por:

$$
\mu_{g}^{t}=\mathrm{C}_{\mu} \rho_{g} \frac{k_{\mathrm{g}}{ }^{2}}{\varepsilon_{\mathrm{g}}} .
$$

Os valores das constantes aplicadas no modelo foram: $\sigma_{k}=0,82 ; \mathrm{C}_{1}=3,4 ; C_{1}^{*}=1,8 ; \mathrm{C}_{2}=$ 4,$2 ; C_{3}=0,8 ; C_{3}^{*}=1,3 ; C_{4}=1,25 ; C_{5}=0,4 ; \sigma_{\varepsilon}=1,0$; $\mathrm{C}_{\varepsilon 1}=1,44 ; \mathrm{C}_{\varepsilon 2}=1,88$.

\section{RESULTADOS E DISCUSSÕES}

Os resultados e discussões apresentam um teste de malha para avaliar a relação da malha numérica frente a perda de carga, a comparação em relação a avaliação da perda de carga obtida na média no plano e na média nos pontos e a validação dos resultados numéricos.

\subsection{Teste de malha}

Quatro malhas distintas (Tabela 1), com uma razão de crescimento dos elementos de $20 \%$ entre elas, foram utilizadas para avaliar a diferença na perda de carga frente o refino de malha. As simulações numéricas do teste de malha foram obtidas para a velocidade na entrada do ciclone em $8 \mathrm{~m} / \mathrm{s}$.

Tabela 1 - Tamanho das malhas utilizada no teste de malha.

\begin{tabular}{cc}
\hline Malha & Número de pontos \\
\hline 1 & 658.742 \\
2 & 1.114 .218 \\
3 & 2.167 .080 \\
4 & 4.291 .277 \\
\hline
\end{tabular}

A Figura 4 apresenta graficamente os valores da perda de carga no ciclone para cada uma das quatro malhas avaliadas. Desses, observou-se que os valores de perda de carga das malhas simuladas encontram-se numa região assintótica, uma vez que estes não variam significativamente com o refinamento da malha. Ainda, com base nos valores 
simulados, conclui-se que não haveria necessidade de utilizar a malha de aproximadamente 4 milhões de pontos, uma vez que a diferença entre os valores da perda de carga da malha 3 para a de 4 é de muito próximo e o custo computacional é no mínimo o dobro. No entanto, devido a um dos objetivos do estudo ser a validação dos resultados de perda de carga, preferiu-se utilizar a malha de maior precisão, ou seja, a malha 4 .

Figura 4 - Teste de malha utilizando velocidade de entrada em $8 \mathrm{~m} / \mathrm{s}$.

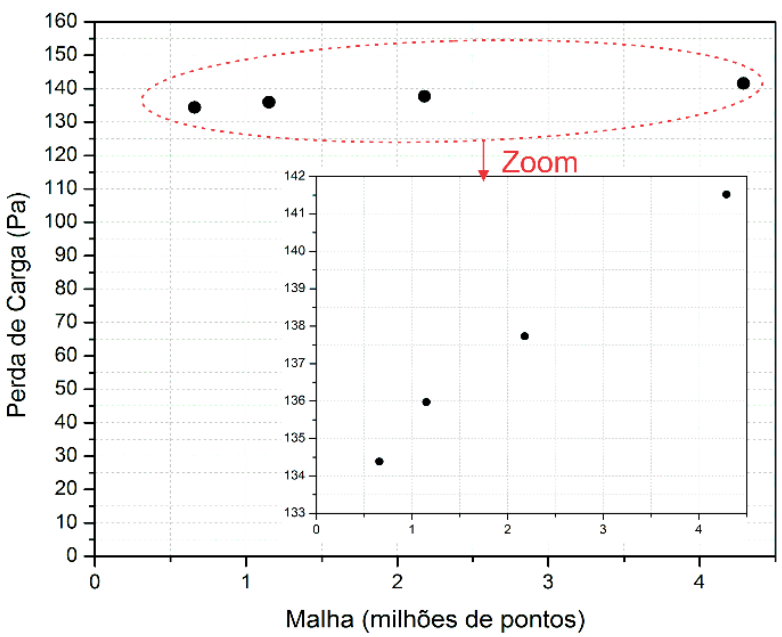

\subsection{Avaliação da perda de carga medida nos pontos e no plano}

Realizou-se uma avaliação de dois métodos de se calcular a perda de carga a partir dos resultados numéricos afim de analisar qual melhor se ajusta aos dados experimentais. Um deles referente a diferença da pressão de entrada e saída medidas em uma média no plano e outro através de uma média nos pontos.

A Figura 5 mostra os campos de pressão na entrada e saída do ciclone resultantes da simulação realizada para a velocidade em 12 $\mathrm{m} / \mathrm{s}$, a localização dos quatro pontos na entrada e saída utilizados para aferir a perda de carga nos pontos, e uma ilustração tridimensional do local da medida de perda de carga experimental. Tal imagem ilustra bem a não uniformidade da pressão no plano de saída e a uniformidade da pressão no plano de entrada do ciclone, também avaliada por Chen e Shi (2006), os quais presumiram que a verdadeira perda de carga é dada pela pressão estática na parede de saída menos a pressão estática na parede de entrada.

Figura 5 - Representação do local de medida da perda de carga simulada quando efetuada nos planos e nos pontos, e ilustração tridimensional do local da medida de perda de carga experimental.
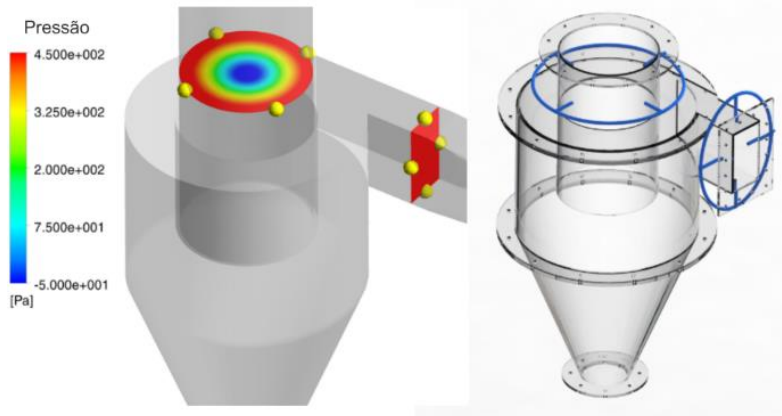

A Tabela 2 apresenta os resultados quantitativos da pressão medida no plano de entrada e saída e a medida na média dos pontos referente a Figura 5.

Tabela 2 - Medida da perda de carga realizada no plano e nos pontos para velocidade de $12 \mathrm{~m} / \mathrm{s}$.

\begin{tabular}{ccc}
\hline Medidas & Plano & Pontos \\
\hline $\begin{array}{c}\text { Pressão na } \\
\text { entrada (Pa) }\end{array}$ & 845,92 & 845,89 \\
$\begin{array}{c}\text { Pressão na } \\
\text { saída (Pa) }\end{array}$ & 331,18 & 522,66 \\
$\begin{array}{c}\text { Perda de } \\
\text { Carga }(\mathrm{Pa})\end{array}$ & 514,74 & 323,23 \\
\hline
\end{tabular}

A partir destes resultados, fica claro que o valor da pressão na região de entrada realizada tanto pela média dos pontos quanto pela média no plano, não tem diferença significativa. Porém, quando avaliado a seção de saída, tem-se uma diferença de 191,48 Pa entre as duas formas de medição. Esta diferença no valor da pressão na saída do ciclone medida através dos dois métodos influência no resultado da perda de carga. 
Tabela 3 - Erro absoluto e relativo da perda de carga medida no plano e nos pontos em relação aos resultados experimentais.

\begin{tabular}{cccccccc}
\hline \multirow{2}{*}{$\mathrm{v}(\mathrm{m} / \mathrm{s})$} & \multicolumn{3}{c}{ Perda de carga (Pa) } & \multicolumn{2}{c}{ Erro absoluto (Pa) } & \multicolumn{2}{c}{ Erro relativo (\%) } \\
& Plano & Pontos & Experimental & Plano & Pontos & Plano & Pontos \\
\hline 8 & 221,0 & 141,5 & 145,1 & $-75,9$ & 3,6 & 52,3 & 2,5 \\
10 & 352,2 & 223,1 & 233,6 & $-118,6$ & 10,5 & 50,8 & 4,5 \\
12 & 514,7 & 323,2 & 343,4 & $-171,4$ & 20,2 & 49,9 & 5,9 \\
14 & 715,3 & 440,2 & 477,2 & $-238,1$ & 37,0 & 49,9 & 7,8 \\
16 & 935,9 & 580,4 & 632,6 & $-303,3$ & 52,2 & 47,9 & 8,3 \\
\hline
\end{tabular}

Portanto, para avaliar qual dos dois métodos (nos pontos ou no plano) é a forma mais adequada de se analisar os resultados numéricos de perda de carga, a Tabela 3 mostra uma comparação das duas formas de medidas da perda de carga em questão, comparados com os resultados de perda de carga medidos experimentalmente na unidade experimental. Esta ainda apresenta os erros absoluto e relativo para cada um dos métodos abordados, levando em conta as cinco velocidades de entrada utilizadas $(8,10,12,14$, e $16 \mathrm{~m} / \mathrm{s})$.

Outra forma de se visualizar a diferença entre as duas formas de medida da perda de carga numérica é através da Figura 6, a qual apresenta um gráfico da perda de carga em relação a velocidade de entrada do ciclone, comparando os resultados obtidos numericamente utilizando os métodos de medida no plano e nos pontos, com os dados obtidos experimentalmente.

Com base na comparação, conclui-se que a perda de carga medida através da média dos pontos se aproxima mais dos resultados obtidos experimentalmente, de maneira que o erro relativo e absoluto são menores quando comparados aos da perda de carga medida pelos planos. Esse comportamento foi observado para todas as velocidades avaliadas. Sendo assim, os experimentos numéricos de perda de carga utilizados para realizar a validação do modelo matemático neste trabalho, foram obtidos através da diferença dos resultados de pressão medidos da média dos pontos de entrada e saída.
Figura 6 - Comparação da perda de carga experimental e numérica medida nos pontos e no plano.

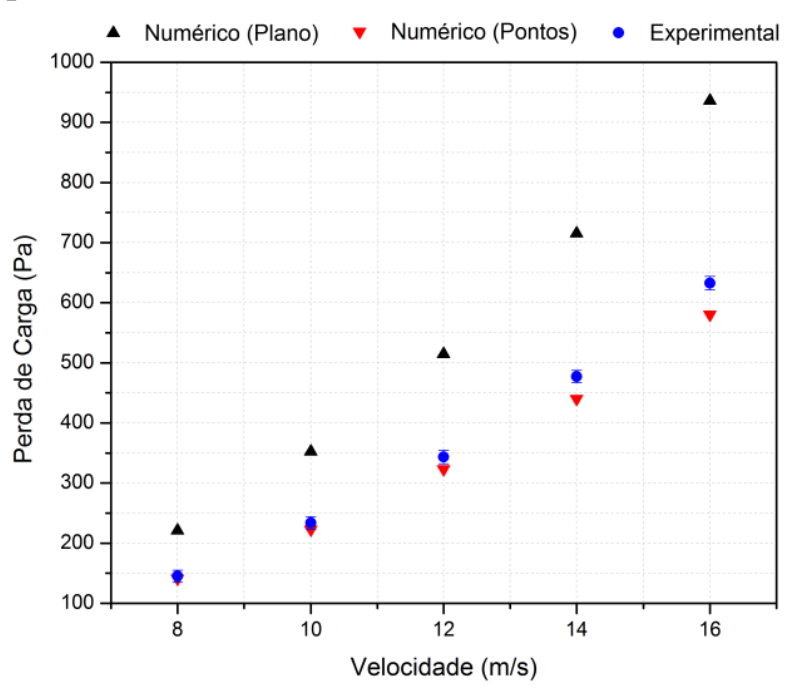

\subsection{Validação da predição da perda de carga}

A validação do modelo de fechamento da turbulência (RSM-SSG) é realizado por comparação dos resultados de perda de carga obtidos nas simulações frente os dados obtidos na unidade experimental. Para melhor ilustrar os resultados de perda de carga experimentais e simulados, gerou-se um gráfico, apresentado na Figura 7, sendo que a barra do erro experimental apresentado, é a soma entre o desvio padrão dos dez experimentos e o erro de precisão do manômetro diferencial utilizado nas medidas; e a barra do erro numérico representa a incerteza numérica calculada através do método GCI (Grid Convergence Index). 
Figura 7 - Comparação dos resultados de perda de carga obtidos das experimentações físicas e numéricas para validação da predição da perda de carga.

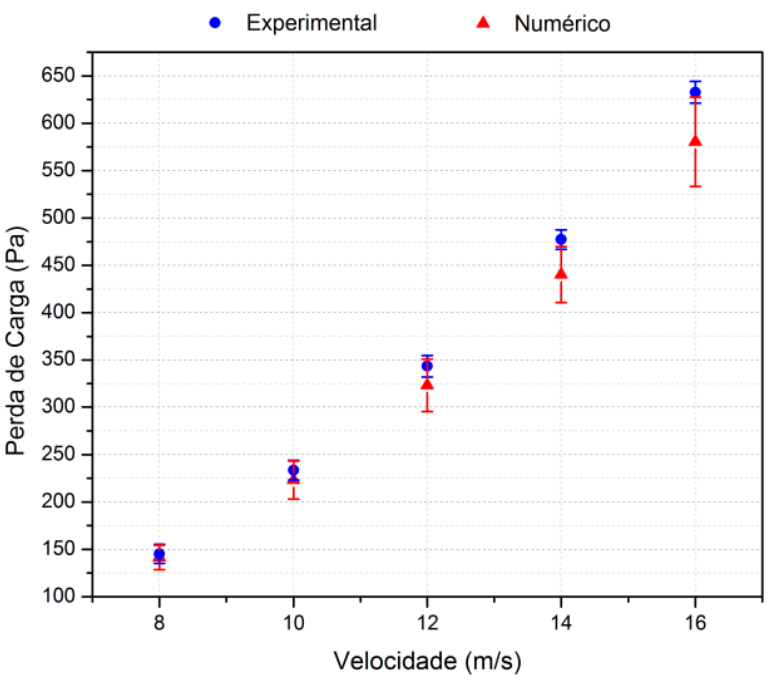

Da comparação dos resultados físicos e numéricos, concluiu-se que a simulação numérica com modelo de turbulência RSMSSG, representou bem os dados experimentais, uma vez que o erro relativo não excedeu os $8,3 \%$ para nenhuma das velocidades estudadas, $\mathrm{e}$ as barras da incerteza experimental e numérica se sobrepuseram.

\section{CONCLUSÕES}

Este estudo avaliou qual a melhor maneira de predizer os resultados de perda de carga numérica em ciclones, se através da diferença de pressão estática entre a entrada e a saída do ciclone, por meio de uma média nos planos ou através de uma média de quatro pontos colocados nas arestas destes planos. Desta análise, a qual foi fundamentada com base nos experimentos físicos, conclui-se que a perda de carga obtida na média dos pontos se mostrou mais adequada para predizer os dados experimentais quando comparadas as obtidas no plano.

Por fim, diante da comparação dos resultados físicos e numéricos pode-se concluir que as equações RANS com o modelo de turbulência RSM-SSG é capaz de predizer a perda de carga em ciclones com uma boa confiabilidade.

\section{NOMENCLATURA}

$\mathrm{r} \equiv$ razão de refinamento;

$\mathrm{q} \equiv$ ordem formal de precisão do algoritmo;

$\mathrm{F}_{s} \equiv$ fator de segurança;

$\mathrm{p} \equiv$ pressão $\left[\mathrm{kg} / \mathrm{ms}^{2}\right]$;

$\mathrm{k} \equiv$ energia cinética turbulenta $\left[\mathrm{m}^{2} / \mathrm{s}^{2}\right]$;

$\mathbf{v} \equiv$ velocidade $[\mathrm{m} / \mathrm{s}]$;

g $\equiv$ aceleração da gravidade $\left[\mathrm{m} / \mathrm{s}^{2}\right]$;

$v \equiv$ viscosidade cinemática molecular $\left[\mathrm{m}^{2} / \mathrm{s}\right]$;

$v^{t} \equiv$ viscosidade cinemática turbulenta $\left[\mathrm{m}^{2} / \mathrm{s}\right]$;

$\mu \equiv$ viscosidade molecular $[\mathrm{kg} \mathrm{m} / \mathrm{s}]$;

$\mu^{t} \equiv$ viscosidade turbulenta $[\mathrm{kg} \mathrm{m} / \mathrm{s}]$;

$\rho \equiv$ densidade $\left[\mathrm{kg} / \mathrm{m}^{3}\right]$

$\varepsilon \equiv$ taxa de dissipação de $\mathrm{k}\left[\mathrm{m}^{2} / \mathrm{s}^{3}\right]$;

$\Upsilon^{*} \equiv$ produção turbulenta $\left[\mathrm{m}^{2} / \mathrm{s}^{3}\right]$;

b $\equiv$ tensor anisotrópico de Reynolds;

S $\equiv$ taxa de deformação [1/s];

$\boldsymbol{\Omega} \equiv$ vorticidade $[1 / \mathrm{s}]$;

$\mathbf{T}^{e f} \equiv$ tensor tensão efetiva $\left[\mathrm{kg} / \mathrm{ms}^{2}\right]$;

$\mathbf{T}^{t} \equiv$ tensor de Reynolds $\left[\mathrm{kg} / \mathrm{ms}^{2}\right]$;

$\mathbf{T} \equiv$ tensor molecular $\left[\mathrm{kg} / \mathrm{ms}^{2}\right]$;

$\mathbf{D}_{\mathbf{T}} \equiv$ termo da difusão turbulenta $\left[\mathrm{kg} / \mathrm{ms}^{3}\right]$;

$\mathbf{D}_{\mathbf{M}} \equiv$ termo da difusão molecular $\left[\mathrm{kg} / \mathrm{ms}^{3}\right]$;

$\mathbf{Y} \equiv$ termo da taxa de produção $\left[\mathrm{kg} / \mathrm{ms}^{3}\right]$;

$\Pi \equiv$ termo da deformação devido a pressão $\left[\mathrm{kg} / \mathrm{ms}^{3}\right]$.

\section{REFERÊNCIAS}

CHEN, J., SHI, M. A. Universal Model to Calculate Cyclone Pressure Drop. Powder Technology, v. 66, p 184-191, 2006.

CHU, K. W., WANG, B., XU, D. L., CHEN, Y. X., YU, A. B. CFD-DEM Simulation of the Gas-Solid Flow in a Cyclone Separator. Chemical Engineering Science, p. 834-847, 2011. 
FREITAS, C. J. The Issue of Numerical Uncertainty. Applied Mathematical Modelling, v. 26, p. 237-248, 2002.

FUNK, P. A. Reducing Cyclone Pressure Drop with Evasés. Powder Technology, v. 272, p. 276-281, 2015.

GIMBUN, J., CHUAH, T. G., L-RAZI, F., CHOONG, T. S. Y. The Influence of Temperature and Inlet Velocity on Cyclone Pressure Drop: a CFD Study. Chimical Engineering and Processing, v. 44, p. 7-12, 2005.

KARIMI, M., AKDOGAN, G., DELLIMORE, K.H., BRADSHAW, S.M., Quantification of Numerical Uncertainty in Computational Fluid Dynamics Modelling of Hydrocyclones. Computers and Chemical Engineering, v. 43, p. 45-54, 2012.

NORILER, D., VEGINI, A. A., SOARES, C., BARROS, A. A. C., MEIER, H. F., MORI, M. A New Role for Reduction in Pressure Drop in Cyclones Using Computational Fluid Dynamics Techniques. Brazilian Journal of Chemical Engineering, Brasil, v. 21, n. 1, p. 93-101, 2004.

ROACHE, P. J. Perspective: A Method for Uniform Reporting of Grid Refinement Studies. Journal of Fluids Engineering, v. 116, p. 405-413, 1994.

SPEZIALE, C. G.; SARKAR, S.; GATSKI, T. B. Modelling the Pressure-Strain Correlation of Turbulence: An Invariant Dynamical Systems Approach. Journal of Fluid Mechanics. v. 227, p. 245-272, 1991.

YUU, S., JOTAKI, T., TOMITA Y., YOSHIDA, K. The Reduction of Pressure Drop due to Dust Loading in a Conventional
Cyclone. Chemical Engineering Science, v. 33, p. 1573-1580, 1978.

\section{AGRADECIMENTOS}

Os autores agradecem a PETROBRAS pelo apoio financeiro sob o termo de cooperação 0050.0070334.11-9, e ao CNPQ processo 310504/2012-0, que tornaram este trabalho possível. 